\title{
Osteopontin is Released From the Heart Into the Coronary Circulation in Patients With a Previous Anterior Wall Myocardial Infarction
}

\author{
Akira Tamura, MD; Maki Shingai, MD; Nobuko Aso, MD; \\ Takayuki Hazuku, MD; Masaru Nasu, MD
}

\begin{abstract}
The present study was designed to clarify whether osteopontin, an extracellular matrix protein, is released from the heart into the coronary circulation in patients with a previous ( $>3$ months) anterior wall myocardial infarction (MI). Using a commercially available enzyme immunoassay kit, plasma concentrations of osteopontin were measured in 30 patients ( 26 men, 4 women; mean age, $61 \pm 12$ years). Blood samples were obtained from the aortic root and coronary sinus. The difference in the plasma concentrations of osteopontin in the aortic root and coronary sinus, which reflects the cardiac production of osteopontin released into the coronary circulation, was compared with the left ventricular ejection fraction (LVEF) and volumes obtained from contrast left ventriculography. Plasma osteopontin concentrations were significantly higher in the coronary sinus than in the aortic root $(672 \pm 446 \mathrm{vs} 610 \pm 398 \mathrm{ng} / \mathrm{ml}, \mathrm{p}=0.02)$. The transcardiac gradient of plasma osteopontin concentration correlated negatively with LVEF $(r=-0.55, p=0.0005)$ and positively with left ventricular $(\mathrm{LV})$ end-diastolic $(r=0.63, p=$ $0.0001)$ and end-systolic volume indexes $(\mathrm{r}=0.79, \mathrm{p}<0.0001)$. This is the first study to show that in patients with a previous anterior wall MI osteopontin is released from the heart into the coronary circulation in proportion to the LV systolic function and volumes, suggesting that this extramatrix protein is associated with post-MI LV remodeling. (Circ J 2003; 67: 742-744)
\end{abstract}

Key Words: Left ventricular remodeling; Myocardial infarction; Osteopontin

$\mathbf{O}$ steopontin is an adhesive glycoprotein containing an arginine-glycine-asparate complex. Although first isolated from mineralized bone matrix, osteopontin can be synthesized by several types of cells, including cardiac myocytes, microvascular endothelial cells and fibroblasts, and it can act as an adhesion molecule, affect cellular function by interacting with integrins, and modulate the expression of inducible nitric oxide synthase $^{2-4}$ It has recently been shown that osteopontin is expressed in the myocardium in acute myocardial infarction (AMI),, 6 pressure overload ${ }^{7,8}$ and heart failure,, 10 but there are no published studies of whether osteopontin is released from the heart in those heart diseases. In the present study, we sought to clarify this question in patients with a previous anterior wall myocardial infarction (MI). In addition, we examined whether the difference in plasma osteopontin concentration between the aortic root and coronary sinus, which reflects the cardiac release of osteopontin, correlates with left ventricular (LV) function and volumes in such patients.

\section{Methods}

\section{Patients}

The study population consisted of 30 patients ( 26 men, 4

(Received January 27, 2003; revised manuscript received May 30, 2003; accepted June 24, 2003)

Second Department of Internal Medicine, Oita Medical University, Oita, Japan

Mailing address: Akira Tamura, MD, Second Department of Internal Medicine, Oita Medical University, Hasama, Oita 879-5593, Japan.

E-mail: akira@oita-med.ac.jp women; mean age, $61 \pm 12$ years) with a previous ( $>3$ months) anterior wall MI; the time since the onset of the AMI ranged from 3 months to 11 years. Exclusion criteria were: recent ( $<3$ months) MI, other heart or lung diseases, bone diseases, collagen diseases, malignant diseases, impaired liver or kidney function (serum creatinine $>1.2 \mathrm{mg} / \mathrm{dl}$ ), and severe trauma including bone fractures or surgical procedures within 6 months of the study. The study protocol was in agreement with the guidelines of the institution's ethics committee, and informed consent was obtained from each patient before the study.

\section{Study Protocol}

A 6 F catheter (Medikit CO, Tokyo, Japan) was placed in the coronary sinus through the femoral vein, and its position was confirmed using injectable contrast dye medium. Next, a 5F pig-tail catheter (Medikit CO) was inserted via the femoral artery and placed in the aortic root. Blood samples were obtained simultaneously from the coronary sinus and the aortic root, after which, coronary angiography and left ventriculography were performed. Left ventriculograms from the $30^{\circ}$ right anterior oblique projection were analyzed for LV ejection fraction (LVEF) by an experienced cardiologist who was unaware of the patients' data. LV volumes were calculated by the area-length method ${ }^{11}$

\section{Measurements of Plasma Osteopontin Concentration}

The plasma osteopontin concentration was measured using a commercially available enzyme immunoassay kit (Immuno-Biological Laboratories CO, Ltd, Gunma, Japan). Intra- and interassay variations were within $2 \%$ and $5 \%$, respectively. 
Table 1 Patient Characteristics

\begin{tabular}{lc}
\hline \hline No. of patients & 30 \\
Age (years) & $61 \pm 12$ \\
M/F & $26 / 4$ \\
Smoking history & $20(67 \%)$ \\
Hypertension & $23(77 \%)$ \\
Diabetes mellitus & $14(47 \%)$ \\
Hyperlipidemia & $25(83 \%)$ \\
Treatment & \\
Angiotensin-converting enzyme inhibitors & $11(37 \%)$ \\
Angiotensin-II type 1 receptor antagonists & $8(27 \%)$ \\
Calcium channel antagonists & $10(33 \%)$ \\
B-blockers & $7(23 \%)$ \\
Furosemide & $8(27 \%)$ \\
Spironolactone & $9(30 \%)$ \\
Digitalis & $2(7 \%)$ \\
LVEF (\%) & $49 \pm 12$ \\
\hline
\end{tabular}

Data are mean \pm SD or number (\%).

$M I$, myocardial infarction; LVEF, left ventricular ejection fraction.

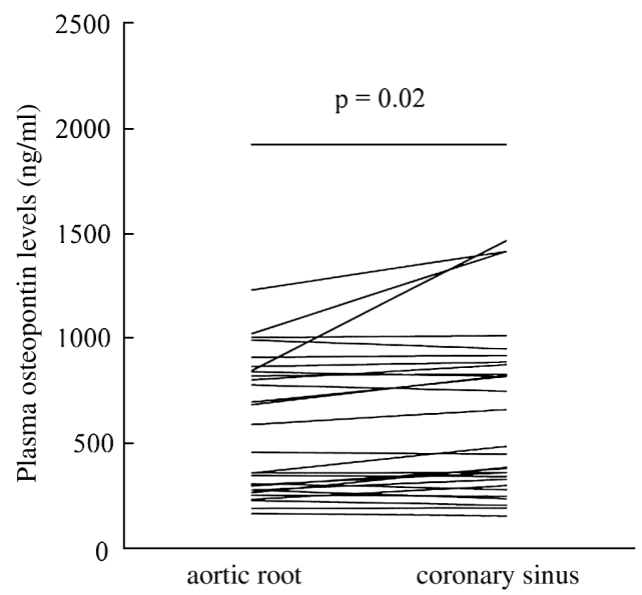

Fig 1. Plasma osteopontin concentrations in the aortic root and coronary sinus.

\section{Statistical Analysis}

Data are expressed as means \pm SD for continuous variables and as numbers (percentages) for categorical variables. Continuous variables were analyzed by the paired test. The Pearson's correlation analysis was performed to estimate correlations between variables. A $p$ value $<0.05$ was considered statistically significant.

\section{Results}

Patient characteristics are shown in Table 1. Of the 30 patients, there was a history of smoking, hypertension, diabetes mellitus, and hyperlipidemia in $20(67 \%), 23$ (77\%), $14(47 \%)$, and 25 patients $(83 \%)$, respectively. Treatment with angiotensin-converting enzyme inhibitors, angiotensin II type 1 receptor antagonists, calcium channel antagonists, $\beta$-blockers, furosemide, spironolactone, and digitalis were currently being administered in $11(37 \%), 8$ (27\%), $10(33 \%), 7$ (23\%), 8 (27\%), 9 (30\%), and 2 patients (7\%), respectively.

The plasma concentration of osteopontin was significantly higher in the coronary sinus than in the aortic root $(672 \pm 446$ vs $610 \pm 398 \mathrm{ng} / \mathrm{ml}, \mathrm{p}=0.02)$ (Fig 1$)$, and the difference in plasma osteopontin concentrations correlated negatively with LVEF $(\mathrm{r}=-0.58, \mathrm{p}=0.0005)$ and positively
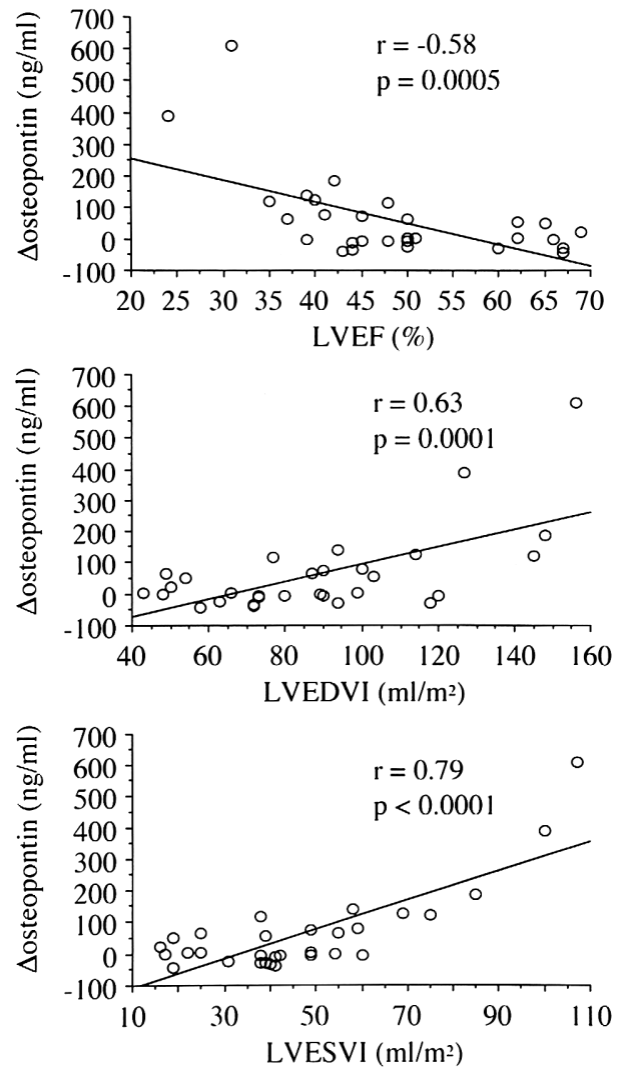

Fig 2. Relationship of the transcardiac gradient of plasma osteopontin to left ventricular ejection fraction (LVEF) and LV end-diastolic and end-systolic volume indexes (LVEDVI and LVESVI, respectively).

with both the LV end-diastolic volume $(\mathrm{r}=0.63, \mathrm{p}=0.0001)$ and LV end-systolic volume indexes $(\mathrm{r}=0.79, \mathrm{p}<0.0001)$ (Fig 2).

\section{Discussion}

To the best of our knowledge, the present study provides the first evidence that osteopontin is released from the heart into the coronary circulation in patients with a previous MI.

Murry et al ${ }^{5}$ demonstrated that osteopontin mRNA and protein are expressed at high levels by macrophages infiltrating the necrotic myocardium of a rat model on days 1 and 2 after thermal injury to the heart and that their expression is diminished by day 4 and is markedly downregulated at 1 and 4 weeks after the myocardial injury. In a human heart with an 8-day-old MI, they also observed abundant expression of osteopontin mRNA and protein in macrophages within the necrotic and granulation tissue, from which they concluded that osteopontin plays an important role in healing after myocardial injury. More recently, in an MI model of osteopontin knockout and wild-type mice, Trueblood et $\mathrm{al}^{6}$ demonstrated that osteopontin expression is increased in the myocardium after MI and that osteopontin knockout mice have greater LV chamber dilation and decreased collagen accumulation after MI compared with wild-type mice. They concluded that osteopontin plays an important role in regulating post-MI LV remodeling, in part by promoting collagen synthesis and accumulation. Thus, the role of osteopontin in MI is being gradually clarified, but to date, it has been unclear whether osteopontin is 
released from the heart into the blood stream of patients with a MI.

In the present study, we measured the plasma concentrations of osteopontin in the aortic root and coronary sinus in patients with a previous anterior wall $\mathrm{MI}$ and found that it was significantly higher in the coronary sinus, indicating that osteopontin is indeed released from the heart into the coronary circulation. Furthermore, the transcardiac gradient of plasma osteopontin was in proportion to LVEF and LV volumes, especially the LV end-systolic volume index. Because the LV end-systolic volume index is currently being used as a marker of post-MI LV remodeling, this result provides additional clinical information that the release of osteopontin into the coronary circulation is associated with post-MI LV remodeling. Taking the results of Trueblood et $\mathrm{al}^{6}$ also into consideration, we presume that the increased expression of osteopontin after an MI protects against LV dilation by promoting collagen synthesis in the infarcted and remote myocardium. An appropriate increase in collagen deposition would be a compensatory response with regard to both infarct repair and the stabilization of myocytes in the remote area after an acute MI. However, in the case of a large MI, the protective effect of osteopontin against LV dilation may be insufficient, and LV dilation may progress despite increased osteopontin expression in the myocardium. Indeed, Stawowy et al found a significant correlation between increased osteopontin immunoreactivity in cardiac myocytes and impaired LV function in patients with heart failure caused by dilated cardiomyopathy,11 although there is not any evidence of this regarding MI.

It has been reported that osteopontin expression in the myocardium diminishes with time 5,6 although the period of observation was limited in those studies. In the present study, we did not find a significant correlation between time elapsed from the onset of MI and transcardiac osteopontin concentrations. It is likely that the expression of osteopontin in the infarcted and remote areas of myocardium lasts for a long time in MI, particularly in a large MI, and the relationship between the time course of the cardiac release of osteopontin into the coronary circulation and post-MI LV remodeling needs to be clarified in future studies.

\section{Study Limitations}

First, the study population was small and therefore, further studies with a large population are needed to confirm the results. Second, the transcardiac osteopontin concentrations in the present patients were not so great when compared with the levels of circulating osteopontin in the aortic root and coronary sinus. Therefore, it cannot be denied that the majority of the circulating osteopontin came from other organs. Third, osteopontin is synthesized at high levels in the smooth muscle cells of plaque, macrophages, and the endothelial cells of the plaque vasa vasorum in human atherosclerosis,${ }^{12-15}$ thus osteopontin may be released from atherosclerotic coronary vessels into the coronary circulation. However, given the close correlation between the transcardiac gradient of plasma osteopontin and LVEF and $\mathrm{LV}$ volumes, it is unlikely that the higher concentration of plasma osteopontin in the coronary sinus was caused by the release of osteopontin from atheromatous coronary vessels. Fourth, angiotensin II has been shown to induce expression of osteopontin ${ }^{7}$ and therefore, angiotensin-converting enzyme inhibitors and angiotensin II type 1 receptor antagonists could reduce the level of circulating osteopontin. However, in the present study, we found no significant difference in transcardiac osteopontin levels between patients with and without these agents, but that lack of difference may have been caused by the small study population. Fifth, it is unclear whether the circulating osteopontin concentration correlates with the tissue concentration of osteopontin in the myocardium and that question needs to be clarified in future studies. Finally, we did not measure the transcardiac osteopontin levels in control subjects.

In conclusion, we have shown for the first time that in patients with a previous anterior wall MI osteopontin is released from the heart into the coronary circulation in proportion to LV systolic function and volumes, which suggests that this extramatrix protein may be associated clinically with post-MI LV remodeling.

\section{References}

1. Franzén A, Heinegård D. Isolation and characterization of two sialoproteins present only in bone calcified matrix. Biochem J 1985; 232: $715-724$.

2. Singh K, Balligand JL, Fischer TA, Smith TW, Kelly RA. Glucocorticoids increase osteopontin expression in cardiac myocytes and microvascular endothelial cells: Role in regulation of inducible nitric oxide synthase. J Biol Chem 1995; 270: 28471-28478.

3. Denhardt DT, Guo X. Osteopontin: A protein with diverse functions. FASEB J 1993; 7: 1475-1482.

4. Giachelli CM, Schwartz SM, Liaw L. Molecular and cellular biology of osteopontin: Potential role in cardiovascular disease. Trends Cardiovasc Med 1995; 5: 88-95.

5. Murry CE, Giachelli CM, Schwartz SM, Vracko R. Macrophages express osteopontin during repair of myocardial necrosis. Am J Pathol 1994; 145: 1450-1462.

6. Trueblood NA, Xie Z, Communal C, Sam F, Ngoy S, Liaw L, et al. Exaggerated left ventricular dilation and reduced collagen deposition after myocardial infarction in mice lacking osteopontin. Circ Res 2001; 88: 1080-1087.

7. Ashizawa N, Graft K, Do YS, Nunohira T, Giachelli CM, Meehan WP, et al. Osteopontin is produced by rat cardiac fibroblasts and mediates AII-induced DNA synthesis and collagen gel contraction. $J$ Clin Invest 1996; 98: 2218-2227.

8. Graft K, Do YS, Ashizawa N, Meehan WP, Giachelli CM, Marboe $\mathrm{CC}$, et al. Myocardial osteopontin expression is associated with left ventricular hypertrophy. Circulation 1997; 96: 3063-3071.

9. Singh K, Sirokman G, Communal C, Robinson KG, Conrad CH, Brooks WW, et al. Myocardial osteopontin expression coincides with the development of heart failure. Hypertension 1999; 33: $663-$ 670.

10. Kennedy JW, Trenholme SE, Kasser IS. Left ventricular volume and mass from single-plane cineangiocardiograms: A comparison of antero-posterior and right anterior oblique methods. Am Heart $J$ 1970; 80: $343-352$.

11. Stawowy P, Blaschke F, Pfautsch P, Goetze S, Lippek F, WollertWulf B, et al. Increased myocardial expression of osteopontin in patients with advanced heart failure. Eur J Heart Fail 2002; 4: 139146.

12. Giachelli CM, Bae N, Almeida M, Denhardt DT, Alpers CE, Schwartz SM. Osteopontin is elevated during neointima formation in rat arteries and is a novel component of human atherosclerotic plaques. J Clin Invest 1993; 92: 1686-1696.

13. Ikeda T, Shirasawa T, Esaki Y, Yoshiki S, Hirokawa K. Osteopontin mRNA is expressed by smooth muscle-derived foam cells in human atherosclerotic lesions of the aorta. J Clin Invest 1993; 92: 28142820.

14. Hirota S, Imakita M, Kohri K, Ito A, Morii E, Adachi S, et al. Expression of osteopontin messenger RNA by macrophages in atherosclerotic plaques: A possible association with calcification. Am J Pathol 1993; 143: $1003-1008$.

15. Pels K, Labinaz M, O'Brien ER. Arterial wall neovascularization: Potential role in atherosclerosis and restsnosis. Jpn Circ J 1997; 61: 893-904. 